\title{
Prevalencia de trematodos y nematodos en asnos comercializados en la Plaza Pecuaria Iscoconga de la provincia de Cajamarca, Perú
}

\author{
Prevalence of trematodes and nematodes in donkeys marketed in the Plaza \\ Pecuaria Iscoconga in the province of Cajamarca, Peru
}

\author{
Luis Vargas-Rocha ${ }^{1,3}$, Esther Malpartida Aquino1, César Murga-Moreno
}

\section{Resumen}

El presente trabajo de investigación tuvo como objetivo determinar la prevalencia de trematodos y nematodos a través de la presencia de huevos en heces de asnos criollos comercializados en la Plaza Pecuaria Iscoconga de Cajamarca, Perú. El trabajo se realizó entre noviembre y diciembre de 2019 con muestras de heces de 73 asnos de ambos sexos. Las muestras fueron analizadas mediante el método de Sedimentación modificada por Rojas y Torrel para el caso de trematodos y el método de Sheather para el caso de nematodos. Todas las muestras fueron positivas al menos a una especie de parásito. Las prevalencias encontradas fueron de Fasciola hepatica $28.8 \pm 10.4 \%$ (21/73), paramphistomidos 0\%; Strongylus spp 53.4 $\pm 11.4 \%$ (39/73), Trichostrongylus spp $46.6 \pm$ $11.4 \%$ (34/73), Nematodirus spp $2.7 \pm 3.7 \%$ (2/73) y Parascaris spp $2.7 \pm 3.7 \%$ (2/73). Se concluye que la prevalencia de parasitosis fue de $100 \%$, correspondiendo $98.6 \%$ a nematodos, $27.4 \%$ a la asociación de $F$. hepatica con nematodos y de $1.4 \%$ a solo $F$. hepatica, sin encontrar asociación estadística significativa entre trematodos y nematodos.

Palabras clave: asnos, Cajamarca, nematodos, prevalencia, trematodos

${ }^{1}$ Escuela Académico Profesional de Medicina Veterinaria, Facultad de Ciencias Veterinarias, Universidad Nacional de Cajamarca, Cajamarca, Perú

${ }^{2}$ Laboratorio de Inmunología e Investigación, Facultad de Ciencias Veterinarias, Universidad Nacional de Cajamarca, Cajamarca, Perú

${ }^{3}$ E-mail: lvargasr17_1@unc.edu.pe; https://orcid.org/0000-0002-8571-6416

Recibido: 23 de septiembre de 2020

Aceptado para publicación: 19 de marzo de 2021

Publicado: 23 de junioi de 2021

CLos autores. Este artículo es publicado por la Rev Inv Vet Perú de la Facultad de Medicina Veterinaria, Universidad Nacional Mayor de San Marcos. Este es un artículo de acceso abierto, distribuido bajo los términos de la licencia Creative Commons Atribución 4.0 Internacional (CC BY 4.0) [https:/ /creativecommons.org/licenses/by/4.0/deed.es] que permite el uso, distribución y reproducción en cualquier medio, siempre que la obra original sea debidamente citada de su fuente original 
The aim of this study was to determine the prevalence of trematodes and nematodes through the presence of eggs in faeces of creole donkeys marketed in the Plaza Pecuaria Iscoconga in Cajamarca, Peru. The work was carried out between November and December 2019 with stool samples from 73 donkeys of both sexes. The samples were analysed using the Sedimentation method modified by Rojas and Torrel for the case of trematodes and the Sheather method for the case of nematodes. All samples were positive for at least one species of parasite. The prevalence found were Fasciola hepatica $28.8 \pm 10.4 \%$ (21/73), paramphistomides 0\%; Strongylus spp $53.4 \pm 11.4 \%$ (39/73), Trichostrongylus spp 46.6 $\pm 11.4 \%$ (34/73), Nematodirus spp $2.7 \pm 3.7 \%$ (2/73) and Parascaris spp $2.7 \pm 3.7 \%(2 / 73)$. It is concluded that the prevalence of parasitosis was $100 \%$; $98.6 \%$ corresponding to nematodes, $27.4 \%$ to the association of $F$. hepatica with nematodes and $1.4 \%$ to only $F$. hepatica, without finding a significant statistical association between trematodes and nematodes.

Key words: Cajamarca, donkeys, nematodes, prevalence, trematodes

\section{INTRODUCCIÓN}

En la región Cajamarca y en toda la sierra peruana predomina una crianza extensiva en materia de producción y explotación animal, en la cual, en un solo predio se pueden encontrar: vacunos, ovinos, caprinos, équidos, porcinos, caninos, etc. Considerando que esta región es endémica en Fasciola hepatica y otros parásitos gastrointestinales, es posible encontrar parásitos que han roto la relación parásito-hospedador específico y se hayan adaptado a otras especies conforme van evolucionando, por lo que es necesario un estudio molecular para determinar género-especie del que se trate (Espinoza et al., 2010; Rodríguez et al., 2014).

Por desconocimiento de las parasitosis en el burro o asno (Equus africanus asinus), los ganaderos no manejan un calendario sanitario aplicado a esta especie, y no se les incluye en un programa de desparasitación. Los productores administran antiparasitarios a los vacunos principalmente, en menor medida a ovinos y ocasionalmente caballos (crianza extensiva), pero no lo hacen a los asnos. Al ser animales que no generan ingresos económicos importantes no se les designa recursos, ya que solo son usados para transporte de carga liviana en distancias cortas, no tienen un costo elevado en el mercado; siendo por el contrario, un gasto importante su dosificación.

Fasciola hepatica ha sido reportada en todos los países del continente americano (Carmona y Fort, 2016). En Perú, las más altas prevalencias de fasciolosis humana y animal son en la Sierra, principalmente en los valles interandinos de Cajamarca, Junín, Cusco y Arequipa, así como en la altiplanicie de la cuenca del Lago Titicaca (Esteban et al., 1999; Marcos et al., 2007). La fasciolosis animal está ampliamente distribuida en 21 de las 24 regiones del Perú, reportándose de manera esporádica o se encuentra ausente en los informes de decomisos en las regiones amazónicas de Madre de Dios, Ucayali y Loreto, donde las condiciones climáticas y ecológicas no favorecerían el desarrollo del ciclo de vida del parásito (Espinoza et al., 2010). 
No se dispone de antecedentes regionales ni nacionales de $F$. hepatica en asnos en el país; sin embargo, se cuenta con un estudio en el Santuario de Burros en Reino Unido donde se reportó 8.5\% (17/200) de animales con presencia de huevos de este trematodo (Svendsen, 1997), mientras que en Turquía se reportaron $2.6 \%$ ( $\mathrm{n}=39$ burros) de infección de F. hepatica (Soykan y Oge, 2012). Asimismo, infecciones por trematodos en burros han sido reportadas por Hasslinger y El-Seify (1996) en Egipto causadas por Gastrodiscus aegyptiacus y Fasciola gigantica, y por Matthee et al. (2004) en Sudáfrica, quienes reportaron 58 especies de nematodos (97\%), 1 cestodo (1.5\%) (Anoplocephalidae: Anoplocephala perfoliata) y 1 especie de trematodo (1.5\%) (Paramphistomidae: Gastrodiscus aegyptiacus) en las muestras de caballos, burros y cebras (Matthee et al., 2004).

De otro lado, la paramphistomidosis es una enfermedad de distribución mundial, reportada con mayor frecuencia en las regiones tropicales y subtropicales (Rangel et al., 2003), siendo las especies de los géneros Paramphistomum, Cotylophoron, Calicophoron, Ugandocotyle, Orthocoelium, Balanorchis y Gastrothylax las principales causantes de la enfermedad en rumiantes (Pinedo et al., 2010; Rojas et al., 2015). En el valle de Cajamarca se reporta Calicophoron microbothrioides (Ortiz et al., 2010).

No se dispone de estudios sobre nematodos gastrointestinales en asnos en Cajamarca ni en Perú. No obstante, en Colombia, Herrera et al. (2016) reportó una frecuencia de parásitos gastrointestinales de $94.4 \%$ en 36 burros de 47 predios, especialmente Trichostrongylus spp (91.7\%), Strongylus spp (68.1\%), Strongyloides westeri $(66.7 \%)$ y Parascaris equorum (9.7\%). De otra parte, Rivero et al. (2018), al analizar muestras de heces de 11 burros en Hidalgo, México, encontraron que el 100\% de los animales presentaron huevos de parásitos gastrointestinales, especialmente
Trichostrongylus spp, Strongylus spp, Trichonema spp, Parascaris equorum y Strongyloide westeri. Es así que el objetivo del presente estudio fue determinar la prevalencia de trematodos y nematodos a través de la presencia de huevos en heces de asnos criollos comercializados en la Plaza Pecuaria Iscoconga de Cajamarca.

\section{Materiales Y MéTodos}

El presente trabajo se llevó a cabo en la Plaza Pecuaria Iscoconga de Cajamarca, provincia de Cajamarca, Perú. La campiña cajamarquina presenta una temperatura media anual de $15.9^{\circ} \mathrm{C}$, precipitación pluvial anual de $537 \mathrm{~mm}$ y humedad relativa anual de $64.5 \%$ (SENAMHI, 2018). Se encuentra ubicada a una altitud de $2750 \mathrm{msnm}$.

Se diseñó un muestreo aleatorio de tipo descriptivo y corte transversal. Entre noviembre y diciembre de 2019 (época lluviosa) se recolectaron muestras de heces de 73 asnos. Para el cálculo del tamaño muestral y al no tener datos de la población de asnos, se usó la fórmula para «n» muestral de una población no conocida (Pita, 2001) con un nivel de confianza al $95 \%$, una proporción esperada al $5 \%$ y un error de muestreo al $5 \%$, el cual quedó definido como 73 muestras fecales.

Las muestras fueron recolectadas de animales adultos ( $>2$ años), independientemente de su sexo. Para la toma de muestras $(100 \mathrm{~g})$, se escogieron las horas de la mañana, se identificaron los asnos que estaban en la plaza y se prestaba atención al momento de la defecación para recolectar las heces en forma inmediata con bolsas de polietileno. Las muestras se almacenaron en una caja de poliestireno expandido (Tecnopor) con hielo en gel y fueron llevadas al Laboratorio de Parasitología Veterinaria de la Facultad de Ciencias Veterinarias de la Universidad Nacional de Cajamarca, para su procesamiento correspondiente en el marco de 48 horas de la colección. 
Para la determinación de nematodos se empleó la técnica de flotación por concentración con solución saturada de azúcar (1 L de agua con $1280 \mathrm{~g}$ de azúcar rubia) a una densidad de 1.20-1.27 mg/ml. Para el caso de trematodos se utilizó el método de Sedimentación modificado por Rojas y Torrel (Torrel y Rojas, 2017). Los géneros de nematodos y trematodos fueron identificados utilizando las características de los huevos descritos por su tamaño y morfología según Fiel et al. (2011) en rumiantes. Para esto, se empleó un microscopio óptico de luz con objetivos de 10 y 40X. La prevalencia de las parasitosis se determinó dividiendo el número de casos positivos entre el total de muestras (animales) y expresado en porcentaje (Thrusfield y Christley, 2018). El análisis estadístico se hizo con la prueba de Chi cuadrado.

\section{Resultados}

La prevalencia total de parasitosis, según la presencia de huevos en heces fue de $100 \%$. En una de las 73 muestras solo se encontró Fasciola, en $98.6 \%$ solo nematodos y en $27.4 \%$ de las muestras se encontró asociaciones entre Fasciola con algún género de nemátodo; no obstante, no se encontró asociación estadística $\left(\mathrm{X}^{2}=3.481\right)$ (Cuadro 1). En el Cuadro 2 se muestra la prevalencia de los cinco géneros de parásitos encontrados en el estudio.

\section{Discusión}

En este estudio se da a conocer los primeros casos de Fasciola hepatica en asnos de Cajamarca y de Perú, encontrándose una prevalencia mayor (28.8\%) a la reportada por Svendsen (1997) en el Reino Unido, quien notificó frecuencias entre 8.5 y $17 \%$. De la misma forma, los resultados de infección por fasciolosis en este estudio, siguen siendo superiores a los encontrados en Turquía por Soykan y Oge (2012) donde la tasa de infección solo fue $2.6 \%$.
Cuadro 1. Prevalencia de trematodos y nematodos según los huevos encontrados en heces de asnos de la Plaza Pecuaria Iscoconga, Cajamarca (2019)

\begin{tabular}{lcc}
\hline \multirow{2}{*}{ Parásitos } & \multicolumn{2}{c}{ Frecuencia } \\
\cline { 2 - 3 } & $\mathrm{n}$ & $\%$ \\
\hline $\begin{array}{l}\text { Fasciola-Strongylus } \\
\text { spp }\end{array}$ & 16 & 21.92 \\
$\begin{array}{l}\text { Fasciola- } \\
\text { Trichostrongylus spp }\end{array}$ & 4 & 5.48 \\
$\begin{array}{l}\text { Fasciola-Nematodirus } \\
\text { spp }\end{array}$ & 1 & 1.37 \\
$\begin{array}{l}\text { Fasciola-Parascaris } \\
\text { spp }\end{array}$ & 1 & 1.37 \\
Solo Fasciola & 1 & 1.37 \\
Solo nematodos & 72 & 98.63 \\
\hline
\end{tabular}

$X^{2}=3.481 ;$ sin significancia estadística $(p>0.05)$

Como lo afirma Espinoza et al. (2010), las más altas prevalencias de fasciolosis humana y animal se presentan en las zonas rurales de los valles andinos de Cajamarca, Junín, Cusco y Arequipa, debido principalmente al pastoreo mixto, aguas estancadas y riegos por inundación con aguas servidas no tratadas, lo cual favorece el desarrollo del hospedador intermediario, caracol del género Lymnaea (viatrix, columella y truncatula).

La prevalencia nula de paramphistomidosis puede deberse a que estos parásitos aún no se han adaptado al tracto gastrointestinal en estos monogástricos, ya que la mucosa es distinta al de los rumiantes; sin embargo, esto no significa que los asnos estén libres de paramphistómidos (Brunser et al., 2013; Rojo y González, 2013). Conocién- 
Cuadro 2. Prevalencia de parásitos gastrointestinales en 73 asnos comercializados en la Plaza Pecuaria Iscoconga, Cajamarca (2019)

\begin{tabular}{lc}
\hline Huevos & $\begin{array}{c}\text { Prevalencia } \\
(\% \pm \text { IC } 95 \%)\end{array}$ \\
\hline Fasciola hepatica & $28.8 \pm 10.4$ \\
Strongylus spp & $53.4 \pm 11.4$ \\
Trichostrongylus spp & $46.6 \pm 11.4$ \\
Nematodirus spp & $2.7 \pm 3.7$ \\
Parascaris spp & $2.7 \pm 3.7$ \\
\hline
\end{tabular}

dose sobre la existencia de Calicophoron microbothrioides en el valle de Cajamarca (Ortiz et al., 2010) será cuestión de tiempo de que estos parásitos puedan adaptarse a los équidos. Por lo pronto, el presente estudio concuerda con el trabajo de Rázuri (2014) quien no encontró paramphistómidos en caballos de Cajamarca.

La alta prevalencia de nematodos en este estudio $(98.6 \%)$ coincide con lo señalado por Herrera et al. (2016) en Colombia; quien encontró 94.4\% (136/144) de muestras positivas y con el estudio de Rivero et al. (2018) en México, con el 100\% de burros $(\mathrm{n}=11)$ con huevos de parásitos gastrointestinales. Matthee et al. (2004) también reportan que el $97 \%$ de los helmintos intestinales en équidos sudafricanos pertenece a 58 especies, siendo solo $1.5 \%$ a una especie de trematodo y otro $1.5 \%$ a una especie de cestodo. Se infiere que la alta prevalencia señalada puede estar ocasionada por la falta de un calendario sanitario que incluya a esta especie, así como a la capacidad adaptativa de los parásitos a las diferentes condiciones fisiológicas y anatómicas de cada especie animal (Rodríguez et al., 2014), y al mal manejo del pastoreo y de las fuentes de agua.

\section{Conclusiones}

- Los asnos en la provincia de Cajamarca están parasitados por helmintos, principalmente por nematodos (Strongylus spp, Trichostrongylus spp, Nematodirus spp y Parascaris spp), seguido del trematodo Fasciola hepatica.

- No se encontraron paramphistomidos.

\section{Agradecimientos}

Los autores agradecen por su compromiso, el tiempo y constante persistencia a los docentes universitarios Dr. M.V. Teófilo Severino Torrel Pajares y Dr. M.V. Juan de Dios Rojas Moncada, de la Cátedra de Parasitología Veterinaria y Enfermedades Parasitarias, Facultad de Ciencias Veterinarias de la Universidad Nacional de Cajamarca.

\section{Literatura Citada}

1. Brunser O, Cruchet S, Gotteland M. 2013. Fisiología gastrointestinal y nutrición. Santiago de Chile: Ed Nestlé Chile. $331 \mathrm{p}$.

2. Carmona C, Tort J. 2017. Fasciolosis en América del Sur: epidemiología y desafíos de control. Rev Helmintol 91: 99109. doi: 10.1017 / S0022149X16000560

3. Espinoza JR, Terashima A, HerreraVelit P, Marcos LA. 2010. Fasciolosis humana y animal en el Perú: impacto en la economía de las zonas endémicas. Rev Perú Med Exp Salud Pública 27: 604-612.

4. Esteban JG, Flores A, Angles R, Mas-Coma S. 1999. High endemicity of human fascioliasis between Lake Titicaca and La Paz valley, Bolivia. Trans R Soc Trop Med Hyg 93: 151-6. doi: 10.1016/s0035-9203(99)90289-4. PMID: 10450437

5. Fiel C, Steffan P, Ferreyra D. 2011. Diagnóstico de las parasitosis más frecuentes de los rumiantes: técnicas de 
diagnóstico e interpretación de resultados. Tandil, Argentina: Abad Benjamin. p 11-34; 100-106.

6. Hasslinger MA, El-Seify MA. 1996. Paramphostomid infestation in equids in Egypt. Berl Munch Tierarztl Wochenschr 109: 224-226.

7. Herrera Y, Vergara J, Ensuncho C, Causil L. 2016. Frecuencia de parásitos gastrointestinales en burros criollos (Equus africanus asinus) en el departamento de Córdoba, Colombia. Rev Colomb Cienc Anim 8: 159-166.

8. Marcos LA, Terashima A, Leguia G, Canales M, Espinoza JR, Gotuzzo E. 2007. La infección por Fasciola hepatica en el Perú: una enfermedad emergente. Rev Gastroenterol 27: 389-396.

9. Matthee S, Krecek RC, McGeoch MA. 2004. A comparison of the intestinal helminth communities of Equidae in Southern Africa. J Parasitol 90: 12631273. doi: $10.1645 / \mathrm{ge}-3353$

10. Ortiz P, Cabrera M, López J, Lenis C, Velásquez L. 2010. Calicophoron microbothrioides. un agente causal de la paramfistomosis en Cajamarca, Perú. En: XXII Congreso Panamericano de Ciencias Veterinarias, Lima.

11. Pinedo R, Chávez A, Casas E, Suárez F, Sánchez N, Huamán H. 2010. Prevalencia de trematodes de la familia Paramphistomatidae en bovinos del distrito de Yurimaguas, provincia de Alto Amazonas, Loreto. Rev Inv Vet Perú 21: 161-167. doi: 10.15381/rivep.v21i2. 132

12. Pita S. 2001. Determinación del tamaño muestral. Unidad de Epidemiología Clínica y Bioestadística. Complexo Hospitalario Juan Canalejo. A Coruña. Cad Aten Primaria 3: 138-14.

13. Rangel-Ruiz LJ, Albores-Brahms ST, Gatriboa-Aguilar J. 2003. Seasonal trends of Paramphistomum cervi in Tabasco, México. Vet Parasit 116: 217 222. doi: 10.1016/j.vetpar.2003.07.002
14. Rázuri B. 2014. Prevalencia de tremátodos en caballos (Equus caballus) en el distrito de Cajamarca. Tesis de Médico Veterinario. Cajamarca: Univ. Nacional de Cajamarca. $32 \mathrm{p}$.

15. Rivero-Perez N, Zaragoza-Bastida A, Vega-Sánchez V, Olave-Leyva I, VegaAngeles J, Peña-Jimenez F. 2018. Identificación de los principales parásitos gastrointestinales en burros del Valle de Tulancingo. AbanicoVet 8: 47-52.

16. Rodríguez J, Pedroso M, Olivares J, Sánchez-Castilleja Y, Arece J. 2014. La interacción hospedero-parásito. Una visión evolutiva. Rev Salud Anim 36: 1-6.

17. Rojas K, Serrano-Martínez E, Tantaleán M, Casas G, Quispe M. 2015. Presencia de Cotylophoron $s p$. en bovinos de la provincia de Moyobamba, Perú. Rev Inv Vet Perú 26: 519-524. doi: 10.15381/rivep.v26i3.11179

18. Rojo C, González E. 2013. Anatomía veterinaria 10. Estómago de los rumiantes. Anatomías externa e interna. Surco gástrico. Posición y relaciones anatómicas. Fijaciones. Reduca Serie Veterinaria 5(2): 28-42.

19. [SENAMHI] Servicio Nacional de Meteorología e Hidrología del Perú..2018. Datos climatológicos de Cajamarca. [Internet]. Disponible en: https:// www.senamhi.gob.pe/

20. Soykan E, Oge H. 2012. [The prevalence of liver trematodes in equines in different cities of Turkey]. Turkiye Parazitol Derg 36: 152-155. doi: 10.5152/ tpd.2012.36. PMID: 23169158

21. Svendsen E. 1997. Manual profesional del burro. $3^{\text {a }}$ ed. Reino Unido: Whittet Books Limited. 57 p.

22. Thrusfield M, Christley R. 2018. Veterinary epidemiology. $4^{\text {th }}$ ed. USA: John Wiley. $68 \mathrm{p}$.

23. Torrel T, Rojas J. 2017. Atlas de parasitología veterinaria. Cajamarca, Perú: Univ. Nacional de Cajamarca. 210 p. 